\title{
On the chaotic properties of the von Foerster-Lasota equation
}

\author{
Zdzisław Brzeźniak • Antoni Leon Dawidowicz
}

Received: 27 September 2011 / Accepted: 18 June 2013 / Published online: 23 October 2013

(C) The Author(s) 2013. This article is published with open access at Springerlink.com

\begin{abstract}
In this article we present a different approach to some of the results published in our recent paper (Brzeźniak and Dawidowicz in Semigroup Forum, 78(1):118-137, 2009). This new approach is based on a deep result from a paper (Ergod. Theory Dyn. Syst. 17(4):793-819, 1997) by Desch Schappacher and Webb.
\end{abstract}

Keywords Chaos $\cdot$ Semigroups of operators $\cdot$ Spectral properties $\cdot$ First-order partial differential equations

\section{Introduction}

The problem of chaotic behavior of solutions of von Foerster partial differential equation was considered by many authors, e.g. the second named author [3, 4], Lasota and Pianigiani [12], Rudnicki [15], Łoskot [14] and Lasota and Szarek [13]. The most useful definition of chaos was given by Devaney [8]. According to Devaney a dynamical system is chaotic if there exists a dense trajectory and the set of periodic points is dense. In papers $[5,6]$ the existence of a periodic solution and a dense trajectory of the equation $\frac{\partial u}{\partial t}+x \frac{\partial u}{\partial x}=\lambda u, t \geq 0, x \in[0,1]$, is proved under the assumption that the parameter $\lambda$ is strictly bigger that 1 . In the paper [2] this result is improved in the sense that the existence of a periodic solutions is proved for any $\lambda>0$. In

Communicated by Markus Haase.

Z. Brzeźniak

Department of Mathematics, University of York, Heslington, York YO10 5DD, UK

e-mail: zdzislaw.brzezniak@york.ac.uk

A.L. Dawidowicz ( $\square)$

Faculty of Mathematics and Computer Science, Jagiellonian University, ul. Łojasiewicza 6, 30-348 Kraków, Poland

e-mail: Antoni.Dawidowicz@im.uj.edu.pl 
the present paper we use a different approach to the same problem. This approach is based on applying some deep results from the paper [7]. Our main result in the most general form, see Theorem 5.5 can be summarized as follows.

Theorem 5.5 Assume, that $p \in[1, \infty)$ and that $h:[0,1] \rightarrow \mathbb{C}$ is a continuous function such that

$$
\exists \delta>0, \quad \lambda>-\frac{1}{p}: \operatorname{Re} h(x)>\lambda, \quad x \in[0, \delta] .
$$

Then the $C_{0}$-semigroup on $L^{p}(0,1)$ generated by the following first order PDE

$$
\frac{\partial u}{\partial t}+x \frac{\partial u}{\partial x}=h(x) u
$$

is chaotic in the sense of Definition 2.2.

In Theorem 5.7 we formulate an analogous result for the Hölder spaces. The proof of both Theorems 5.5 and 5.7 is based on applying similar results when function $h$ is constant, see Theorem in Sect. 4 An anonymous referee has asked whether our method can be used to get alternative prove of the main result from the paper [16] by Takeo. Our results from Sect. 5 not only give an affirmative answer to this question but in fact are stronger than the corresponding ones from [16].

In a forthcoming publication we plan to generalize results presented he to problems in multidimensional domains.

Notation By $\mathbb{C}$, respectively $\mathbb{R}$, we will denote the set of complex, resp. real, numbers. By $\mathbb{C}^{*}$, respectively $\mathbb{R}^{*}$, we will denote the set of nonzero complex, resp. real, numbers. A subset $Z$ of $\mathbb{C}^{*}$ will be called bounded if there exists $r>0$ such that $r \leq|z| \leq \frac{1}{r}$ for all $z \in Z$. A function $f$ with values in $\mathbb{C}^{*}$ will be called bounded, if the range of $f$ is a bounded subset of $\mathbb{C}^{*}$.

\section{Formulation of the problem}

Let consider the following differential equation

$$
\frac{\partial u}{\partial t}+x \frac{\partial u}{\partial x}=\lambda u, \quad t \geq 0, x \in[0,1],
$$

together with the initial condition

$$
u(0, x)=v(x), \quad x \in[0,1] .
$$

The paper [2] contains results about stability and chaos of the dynamical systems generated by this equation in various Banach spaces of functions on the interval $[0,1]$. In particular we showed that for each such a space there is a threshold $\lambda_{c}$ such that the semigroup generated by (2.1) is chaotic or asymptotically stable depending whether $\lambda>\lambda_{c}$ or $\lambda \leq \lambda_{c}$. Our approach to the chaos was based on a classical Avez method 
used earlier by Lasota and Pianigiani [12] and the second named authour [4] to study the problem of the existence of chaos in the space of Lipschitz functions. The main aim of the present paper is to present an alternative proof of our results from [2] based on the aforementioned paper [7] by Desch, Schappacher and Webb. In that paper the authours proved the following fundamental result.

Theorem 2.1 Let $X$ be a separable complex Banach space and let $A$ be the infinitesimal generator of a strongly continuous semigroup $\{T(t)\}_{t \geq 0}$ on $X$. Let $U$ be open subset of the point spectrum of $A$ which intersects the imaginary axis $i \mathbb{R}$. For each $\gamma \in U$ let $u_{\gamma}$ be a nonzero eigenvector of $A$, i.e. $A u_{\gamma}=\gamma u_{\gamma}$. For each $\Phi \in X^{*}$, let us define a function $F_{\Phi}: U \ni \gamma \mapsto\left\langle\Phi, u_{\gamma}\right\rangle \in \mathbb{C}$. Assume, that for each $\Phi \in X^{*} \backslash\{0\}$, the function $F_{\Phi}$ is analytic and it does not vanish identically on $U$. Then the semigroup $\{T(t)\}_{t \geq 0}$ is chaotic on $X$.

Before formulating the theorem we recall the definition of chaos formulated in [8, p. 117], see also Appendix A of [2].

Definition 2.2 Let $\varphi=\left(\varphi_{t}\right)_{t \geq 0}$ be a $C_{0}$-semigroup of bounded linear operators on a normed vector space $(X,|\cdot|)$. It is said that $\varphi$ is chaotic iff it satisfies the following three properties:

(i) $\varphi$ is transitive, i.e. for all $x, y \in X$ and $\varepsilon>0$ there exists $z \in X$ such that $\operatorname{dist}\left(x, \gamma^{+}(z)\right)<\varepsilon$ and $\operatorname{dist}\left(y, \gamma^{+}(z)\right)<\varepsilon$, where $\gamma^{+}(z)$ is the positive orbit of $\varphi$ through $z$, i.e. $\gamma^{+}(z):=\left\{\varphi_{t}(z): t \geq 0\right\}$, see e.g. [10].

(ii) $\varphi$ depends sensitively on initial data, i.e. there exists $\beta>0$ such that for all $x \in X$ and $\varepsilon>0$ there exists $y \in B(x, \varepsilon)$ and $t>0$ such that $\left|\varphi_{t}(x)-\varphi_{t}(y)\right| \geq \beta$.

(iii) the periodic points of $\phi$ are dense in $X$.

Let us now assume that $X$ is a certain Banach space of functions defined on the interval $[0,1]$ and an operator $A$ defined informally by the following formula

$$
A u=\lambda u-x \frac{\partial u}{\partial x} .
$$

For a given complex number $\gamma$ let us define a function $u_{\gamma}$ by the following equality

$$
u_{\gamma}(x)=x^{\lambda-\gamma}, \quad x \in(0,1]
$$

The function $u_{\gamma}$ is a candidate for the eigenvector $u_{\gamma}$ from Theorem 2.1. Clearly if $u_{\gamma} \in X$ and $\Phi \in X^{*}$, then the function $F_{\Phi}$ introduced in Theorem 2.1 has the following representation

$$
F_{\Phi}(\gamma)=\sum_{k=0}^{\infty} \frac{1}{k !} \Phi\left(f_{k}\right) \gamma^{k}, \quad \gamma \in \mathbb{C},
$$

where, for $k \in \mathbb{N}$,

$$
f_{k}(x)=x^{\lambda}(-\ln x)^{k}, \quad x \in(0,1] .
$$


Hence to prove the chaos property of the semigroup generated by the operator $A$ (whose domain is denoted by $D(A)$ ), it is sufficient to show that the following three conditions are satisfied.

(i) The set $\left\{\gamma \in \mathbb{C}: u_{\gamma} \in D(A)\right\}$ contains an open set with nonempty intersection with the imaginary axis.

(ii) The set $\left\{f_{k}: k=0,1,2, \ldots\right\}$ is linearly dense in $X$.

(iii)

$$
\limsup _{k \rightarrow \infty} \sqrt[k]{\frac{\left|f_{k}\right|}{k !}}<\infty .
$$

In the following sections we will show how this program will be realized in the cases when $X=L^{p}(0,1)$ or $X=C_{0}^{\alpha}([0,1]), \alpha \in(0,1)$.

\section{The case of the space $L^{p}$}

In the first we consider our problem in the space $L^{p}(0,1), p \in[1, \infty)$. As in [17] it can be shown that in this case

$$
\begin{gathered}
D(A)=\left\{u \in L^{p}(0,1): u\right. \text { is almost everywhere differentiable } \\
\text { and } \left.\int_{0}^{1}\left|x u^{\prime}(x)\right|^{p} d x<\infty\right\} .
\end{gathered}
$$

The function $u_{\gamma}$ defined by formula (2.3) is an element of the space $L^{p}(0,1)$ if and only if

$$
\int_{0}^{1}|u(x)|^{p} d x=\int_{0}^{1} x^{(\lambda-\operatorname{Re}(\gamma)) p} d x<\infty .
$$

Since $\int_{0}^{1} x^{r} d x$ is finite iff $r>-1$, we infer that $u_{\gamma} \in L^{p}$ iff $(\lambda-\operatorname{Re}(\gamma)) p>-1$. The last condition is equivalent to the following one

$$
\operatorname{Re} \gamma<\lambda+\frac{1}{p}
$$

Moreover, if the condition (3.2) is satisfied, then the function $u_{\gamma}$ is differentiable on $(0,1]$ and $u_{\gamma}^{\prime}(x)=(\lambda-\operatorname{Re}(\gamma)) x^{(\lambda-\operatorname{Re}(\gamma)-1)}$. Therefore $x u_{\gamma}^{\prime}(x)=(\lambda-$ $\operatorname{Re}(\gamma)) x^{(\lambda-\operatorname{Re}(\gamma))}$ and in view of the equality (3.1) we infer that $u_{\gamma} \in D(A)$. Hence we proved that $u_{\gamma} \in D(A)$ iff condition (3.2) is satisfied.

We are ready to state the main result of this section.

Theorem 3.1 For $\lambda>-\frac{1}{p}$ the semigroup $\{T(t)\}$ on the space $L^{p}$ is chaotic.

Proof of Theorem 3.1 Let us fix $\lambda \in \mathbb{R}$ such that $\lambda>-\frac{1}{p}$.

Then the condition (i) is an obvious consequence of condition (3.2). 
Next we shall show that the set of functions of the form

$$
\left\{(0,1] \ni x \mapsto x^{\lambda}(-\ln x)^{n}\right\}, \quad n \in \mathbb{N}
$$

is linearly dense in $L^{p}((0,1])$.

We begin the proof by observing, that each of these functions belong to $L^{p}(0,1]$. Indeed, since $\lambda p+1>0$ we have

$$
\int_{0}^{1} x^{\lambda p}(-\ln x)^{n p} d x=\int_{0}^{\infty} e^{-s \lambda p} s^{n p} e^{-s} d s=\int_{0}^{\infty} e^{-(\lambda p+1) s} s^{n p} d s<\infty .
$$

For a function $f \in L^{p}(0,1)$ define a function If by the formula

$$
\text { If }(x)=f\left(e^{-x}\right) e^{-\frac{x}{p}}, \quad x \in(0, \infty) .
$$

One can easily show that $I f$ belongs to $L^{p}(0, \infty)$ and that the map $I: L^{p}(0,1) \rightarrow$ $L^{p}(0, \infty)$ is an isometric isomorphism.

Moreover, the image by $I$ of the set defined in (3.3) is equal to the set

$$
\left\{y \mapsto y^{n} e^{-\left(\lambda+\frac{1}{p}\right) y}: n \in \mathbb{N}\right\},
$$

which, by [11, Proposition on p. 160], is linearly dense in $L^{p}(0, \infty)$. This proves that the condition (ii) holds.

It remains to show the condition (iii) holds as well. For this aim we notice that

$$
\left\|f_{k}\right\|_{L^{p}}=\left(\int_{0}^{1} x^{\lambda p}(-\ln x)^{k p} d x\right)^{\frac{1}{p}}
$$

Using the change of variables $z=-(\lambda p+1) \ln x$ we obtain the following equality, for $k \in \mathbb{N}$,

$$
\int_{0}^{1} x^{\lambda p}(-\ln x)^{k p} d x=\frac{1}{(\lambda p+1)^{k p+1}} \int_{0}^{\infty} z^{k p} e^{-z} d z=\frac{1}{(\lambda p+1)^{k p+1}} \Gamma(k p+1) .
$$

Hence, we have

$$
\left\|f_{k}\right\|_{L^{p}}=\left(\frac{1}{(\lambda p-1)^{k p+1}} \Gamma(k p+1)\right)^{\frac{1}{p}}, \quad k \in \mathbb{N}
$$

and consequently

$$
\sqrt[k]{\frac{\left\|f_{k}\right\|_{L^{p}}}{k !}}=\frac{1}{(\lambda p+1)^{1+\frac{1}{k p}}} \frac{\Gamma(k p+1)^{\frac{1}{k p}}}{(k !)^{\frac{1}{k}}}=\frac{1}{(\lambda p+1)^{1+\frac{1}{k p}}} \frac{\Gamma(k p+1)^{\frac{1}{k p}}}{\Gamma(k+1)^{\frac{1}{k}}} .
$$

Therefore

$$
\lim _{k \rightarrow \infty} \sqrt[k]{\frac{\left\|f_{k}\right\|_{L^{p}}}{k !}}=\frac{p}{\lambda p+1}<\infty,
$$

which concludes the proof of condition (iii). In view of Theorem 2.1, Theorem 3.1 follows. 


\section{The case of the Hölder spaces}

Let us assume that $\alpha \in(0,1]$. By $C^{\alpha}([0,1])$ we denote the Banach space of all complex valued Hölder continues functions with exponent $\alpha$ equipped with the standard norm:

$$
|v|_{C^{\alpha}([0,1])}=|v|_{L^{\infty}(0,1)}+H_{1, \alpha}(v),
$$

where

$$
H_{r, \alpha}(v)=\sup \left\{\frac{|v(x)-v(y)|}{|x-y|^{\alpha}}: x, y \in[0,1], 0<|x-y|<r\right\} .
$$

By $c^{\alpha}([0,1])$ we denote the closed subspace of the Banach space $C^{\alpha}([0,1])$ consisting of those $v \in C^{\alpha}([0,1])$ which satisfy

$$
\lim _{r \searrow 0} H_{r, \alpha}(v)=0 .
$$

As in [2] we put

$$
\mathrm{V}_{\alpha}=v \in c^{\alpha}([0,1]) \text { which satisfy the conditions: } v(0)=0 .
$$

Obviously, $\mathrm{V}_{\alpha}$ is a closed subspace of $c^{\alpha}([0,1])$.

The restriction of the function $H_{1, \alpha}$ to the space $\mathrm{V}_{\alpha}$ is a norm on that space which is equivalent to the norm induced by the original norm induced from the space $c^{\alpha}([0,1])$.

As in [17] it can be shown that in this case

$$
D(A)=\left\{u \in \mathrm{V}_{\alpha}: u \in C^{1}([0,1]) \text { and } u^{\prime}(\cdot) \in \mathrm{V}_{\alpha}\right\} .
$$

Before we formulate the main result in this section let us introduce some auxiliary notation. We begin with recalling that the Sobolev space ${ }_{0} W^{1, p}([0,1])$ is the Banach space of all $f \in L^{p}(0,1)$ such that the weak derivative $f^{\prime}$ belongs to $L^{p}(0,1)$ and $f(0)=0$. Alternatively, ${ }_{0} W^{1, p}([0,1])$ is the Banach space of all absolutely continuous functions $f:[0,1] \rightarrow \mathbb{R}$ such that $f(0)=0$ and the derivative $f^{\prime}$ (which exists as $f$ is differentiable almost everywhere), belongs to $L^{p}(0,1)$. The classical norm on ${ }_{0} W^{1, p}([0,1])$, the one inherited from $W^{1, p}([0,1])$, is equivalent to the following one

$$
\|f\|_{0} W^{1, p}:=\int_{0}^{1}\left|f^{\prime}(x)\right|^{p} d x .
$$

Moreover, the space ${ }_{0} W^{1, p}([0,1])$ is isometrically isomorphic to the space $L^{p}([0,1])$ and the corresponding isomorphism is the derivative map

$$
D:{ }_{0} W^{1, p}([0,1]) \ni f \mapsto f^{\prime} \in L^{p}([0,1]) .
$$

Let us recall that according to the Morrey's inequality, see [9, Sect. 5.6, Theorem 5], if $1-\alpha>\frac{1}{p}$, then ${ }_{0} W^{1, p}([0,1])$ is continuously embedded into the space $V_{\alpha}$. Moreover, ${ }_{0} W^{1, p}([0,1])$ is a dense subset of $V_{\alpha}$. Indeed, if $u \in \mathrm{V}_{\alpha}$ then we can define 
a piece-wise linear function on $u_{n}:[0,1] \rightarrow \mathbb{R}$ such that $u_{n}\left(\frac{k}{n}\right)=u\left(\frac{k}{n}\right)$ for every $k=0, \ldots, n$. It is easy to notice, that

$$
\frac{\left|v_{n}(x)-v_{n}(y)\right|}{|x-y|^{\alpha}} \leq 4 \sup _{0<|\xi-\eta| \leq \frac{1}{n}} \frac{|u(\xi)-u(\eta)|}{|\xi-\eta|^{\alpha}},
$$

where $v_{n}=u-u_{n}$. Since the functions $u_{n}$ belong to $W_{1, p}$ for every $p \geq 1$ and by (4.2) $H_{1, \alpha}\left(u_{n}-u\right) \rightarrow 0$, the proof of our claim is complete.

Theorem 4.1 If $\lambda>\alpha$ then the semigroup $\{T(t)\}$ is chaotic in the space $\mathrm{V}_{\alpha}$.

Proof Analogously to proof of the Theorem 3.1 we notice, that the function $u_{\gamma}$ defined by (2.3) belongs to $\mathrm{V}_{\alpha}$ if and only if $\operatorname{Re}(\lambda-\gamma)>\alpha$. Since the function $[0,1] \ni x \mapsto x^{\rho}$ belongs to $\mathrm{V}_{\alpha}$ if and only if $\operatorname{Re} \rho>\alpha$ we infer that $u_{\gamma} \in \mathrm{V}_{\alpha}$ for $\operatorname{Re} \gamma<\lambda-\alpha$. Moreover, by (4.3) it follows that in this case $u_{\gamma} \in D(A)$ as well.

Let us now fix $\lambda \in \mathbb{R}$ such that $\lambda>\alpha$. Let us choose an auxiliary number $p$ such that $\lambda-1>\frac{1}{p}$.

First we shall prove, that the set of the functions

$$
\left\{[0,1] \ni x \mapsto x^{\lambda}(-\ln x)^{n}: n \in \mathbb{N}\right\}
$$

is linearly dense in $V_{\alpha}$.

Let us define a function $g_{n}^{\lambda}$ by

$$
g_{n}^{\lambda}(x)=x^{\lambda}(-\ln x)^{n}, \quad x \in(0,1] .
$$

We can notice, that

$$
D g_{n}^{\lambda}=\lambda g_{n}^{\lambda-1}-n g_{n-1}^{\lambda-1} \text {. }
$$

Since $D:{ }_{0} W^{1, p}([0,1]) \rightarrow L^{p}([0,1])$ is an isomorphism and the set $\left\{g_{n}^{\lambda-1}: n \in \mathbb{N}\right\}$ is linearly dense in $L^{p}([0,1])$, we infer that the set $\left\{g_{n}^{\lambda}: n \in \mathbb{N}\right\}$ is linearly dense in $_{0} W^{1, p}([0,1])$. Since ${ }_{0} W^{1, p}([0,1])$ is dense in $V_{\alpha}$, it follows, that the set $\left\{g_{n}^{\lambda}: n \in \mathbb{N}\right\}$ is linearly dense in $\mathrm{V}_{\alpha}$ as well. This completes the proof of condition (ii).

Now, we shall prove, that

$$
\limsup _{k \rightarrow \infty} \sqrt[k]{\frac{\left\|f_{k}\right\|}{k !}}<\infty
$$

where $\|\cdot\|=\|\cdot\| \mathrm{v}_{\alpha}$. Clearly

$$
f_{k}(x)^{\frac{1}{\alpha}}=x^{\frac{\lambda}{\alpha}}(-\ln x)^{\frac{k}{\alpha}}, \quad x \in(0,1] .
$$

It is easy, to notice, that

$$
\left|\frac{d}{d x}\left(f_{k}(x)^{\frac{1}{\alpha}}\right)\right| \leq \frac{\lambda+k}{\alpha} \exp \left(-k\left(\frac{1}{\alpha}-\frac{1}{\lambda}\right)\right)\left(\frac{k}{\lambda}\right)^{\frac{k}{\alpha}}, \quad x \in(0,1] .
$$


Hence, for all $x, y \in[0,1]$

$$
\left|f_{k}(x)^{\frac{1}{\alpha}}-f_{k}(y)^{\frac{1}{\alpha}}\right| \leq \frac{\lambda+k}{\alpha} \exp \left(-k\left(\frac{1}{\alpha}-\frac{1}{\lambda}\right)\right)\left(\frac{k}{\lambda}\right)^{\frac{k}{\alpha}}|x-y|
$$

Since $\alpha<1$ the function $x \mapsto x^{\frac{1}{\alpha}}$ is convex and vanishing in 0 ,

$$
\left|f_{k}(x)-f_{k}(y)\right|^{\frac{1}{\alpha}} \leq\left|f_{k}(x)^{\frac{1}{\alpha}}-f_{k}(y)^{\frac{1}{\alpha}}\right|, \quad x, y \in[0,1] .
$$

Therefore, for all $x, y \in[0,1]$,

$$
\left|f_{k}(x)-f_{k}(y)\right| \leq\left(\frac{\lambda+k}{\alpha}\right)^{\alpha} \exp \left(-k\left(1-\frac{\alpha}{\lambda}\right)\right)\left(\frac{k}{\lambda}\right)^{k}|x-y|^{\alpha} .
$$

Hence

$$
\left\|f_{k}\right\|_{V_{\alpha}} \leq\left(\frac{\lambda+k}{\alpha}\right)^{\alpha} \exp \left(-k\left(1-\frac{\alpha}{\lambda}\right)\right)\left(\frac{k}{\lambda}\right)^{k}
$$

and thus

$$
\sqrt[k]{\frac{\left\|f_{k}\right\| V_{\alpha}}{k !}} \leq \sqrt[k]{\left(\frac{\lambda+k}{\alpha}\right)^{\alpha}} \exp \left(\frac{\alpha}{\lambda}-1\right) \sqrt[k]{\frac{1}{\lambda}} \frac{k}{\sqrt[k]{k !}}
$$

from which it follows that

$$
\limsup _{k \rightarrow \infty} \sqrt[k]{\frac{\left\|f_{k}\right\|_{V_{\alpha}}}{k !}} \leq e^{\frac{\alpha}{\lambda}}<\infty
$$

This completes the proof.

\section{The case of non-constant function $h$}

In the paper [16] a theorem similar to our Theorem 3.1 has been proved but with the constant $\lambda$ being replaced by a continuous function $h:[0,1] \rightarrow \mathbb{C}$ satisfying the following two conditions.

1. There exists a number $\delta>0$ such that $\operatorname{Re} h(x) \geq 0$ for $x \in[0, \delta]$.

2. $\operatorname{Re} h(x)>-\frac{1}{p}, x \in[0,1]$.

Takeo's result is different from ours, because for $\lambda<0$, a constant function $h=\lambda$ does not satisfy condition (1). We will prove a result stronger than Theorem 3.5 in [16]. For this purpose let us consider the following first order partial differential equation.

$$
\frac{\partial u}{\partial t}+x \frac{\partial u}{\partial x}=h(x) u
$$

At first we will show that the asymptotic behavior of the $C_{0}$-semigroup generated by equation (5.1) depends only on the behavior of the function $h$ in the neighborhood of 0 . To be precise we will prove the following. 
Proposition 5.1 Let $X$ be a Banach space consisting of complex valued functions defined on the interval $[0,1]$. Assume that $h$ and $\widehat{h}$ the two continuous $\mathbb{C}$-valued functions defined on $[0,1]$ such that for some $\delta>0$

$$
h(x)=\widehat{h}(x), \quad \text { for every } x \in[0, \delta] .
$$

Consider also the following differential equation

$$
\frac{\partial u}{\partial t}+x \frac{\partial u}{\partial x}=\widehat{h}(x) u
$$

Assume, that both equations (5.1) and (5.2) generate $C_{0}$-semigroups on $X$ denoted by, respectively, $\left\{T_{t}\right\}_{t \geq 0}$ and $\left\{\widehat{T}_{t}\right\}_{t \geq 0}$.

Then, there exist such $t_{0}>0$ and a continuous function $g:[0,1] \rightarrow \mathbb{C}^{*}$, such that

$$
g(x)=1 \quad \text { for } x \in\left[0, \mathrm{e}^{-t_{0}}\right]
$$

and

$$
T_{t} u=g \widehat{T}_{t} u \quad \text { for every } t \geq t_{0} .
$$

Proof Let us begin with the observation that for every $u \in X$ we have

$$
T_{t} u(x)=\exp \left(\int_{-t}^{0} h\left(x \mathrm{e}^{s}\right) d s\right) u\left(x \mathrm{e}^{-t}\right), \quad x \in[0,1]
$$

and

$$
\widehat{T}_{t} u(x)=\exp \left(\int_{-t}^{0} \widehat{h}\left(x \mathrm{e}^{s}\right) d s\right) u\left(x \mathrm{e}^{-t}\right), \quad x \in[0,1] .
$$

Let us next choose $t_{0}>0$ such that $\mathrm{e}^{-t_{0}}<\delta$. Let us then take $t>t_{0}$. Then we have the following train of equalities for every $x \in[0,1]$.

$$
\begin{aligned}
T_{t} u(x)= & \exp \left(\int_{-t}^{0} h\left(x \mathrm{e}^{s}\right) d s\right) u\left(x \mathrm{e}^{-t}\right) \\
= & \exp \left(\int_{-t}^{-t_{0}} h\left(x \mathrm{e}^{s}\right) d s+\int_{-t_{0}}^{1} h\left(x \mathrm{e}^{s}\right) d s\right) u\left(x \mathrm{e}^{-t}\right) \\
= & \exp \left(\int_{-t}^{-t_{0}} \widehat{h}\left(x \mathrm{e}^{s}\right) d s+\int_{-t_{0}}^{0} h\left(x \mathrm{e}^{s}\right) d s\right) u\left(x \mathrm{e}^{-t}\right) \\
= & \exp \left(\int_{-t}^{-t_{0}} \widehat{h}\left(x \mathrm{e}^{s}\right) d s+\int_{-t_{0}}^{0} \widehat{h}\left(x \mathrm{e}^{s}\right) d s-\int_{-t_{0}}^{1} \widehat{h}\left(x \mathrm{e}^{s}\right) d s\right. \\
& \left.+\int_{-t_{0}}^{1} h\left(x \mathrm{e}^{s}\right) d s\right) u\left(x \mathrm{e}^{-t}\right)
\end{aligned}
$$




$$
\begin{aligned}
& =\exp \left(\int_{-t_{0}}^{0}\left(h\left(x \mathrm{e}^{s}\right)-\widehat{h}\left(x \mathrm{e}^{s}\right)\right) d s\right) \exp \left(\int_{-t}^{0} \widehat{h}\left(x \mathrm{e}^{s}\right) d s\right) u\left(x \mathrm{e}^{-t}\right) \\
& =\exp \left(\int_{-t_{0}}^{0}\left(h\left(x \mathrm{e}^{s}\right)-\widehat{h}\left(x \mathrm{e}^{s}\right)\right) d s\right) \widehat{T}_{t} u(x) .
\end{aligned}
$$

This implies that with a function $g$ defined by the formula

$$
g(x)=\exp \left(\int_{-t_{0}}^{0}\left(h\left(x \mathrm{e}^{s}\right)-\widehat{h}\left(x \mathrm{e}^{s}\right)\right) d s\right), \quad x \in[0,1] .
$$

we have, for every $t>t_{0}$,

$$
T_{t} u(x)=g(x) \widehat{T}_{t} u(x), \quad x \in[0,1] .
$$

The proof is complete.

Remark 5.2 If the operation of multiplication by the function $g$ is an isomorphism of $X$, then the chaos property of the system generated by (5.1) is equivalent to the chaos property of the system generated by (5.2).

Justification of the claim made in Remark 5.2 Let us denote by $\mathfrak{C}$ the linear operator of multiplication by $g$. It follows from formulae (5.5) and (5.6) that the functions $T_{t} u$ and $\widehat{T}_{t} u$ depend only on the values of $u$ on the interval $\left[0, \mathrm{e}^{-t}\right]$. Therefore, assumption (5.3) implies that $g(x) u(x)=u(x)$, on this interval, provided that $t>t_{0}$. Hence, we infer that $T_{t} \mathfrak{C}=T_{t}$ for $t>t_{0}$. On the other hand, in view of Proposition 5.1, from assumption (5.3) implies that $T_{t} \mathfrak{C}=\mathfrak{C} \widehat{T}_{t}$.

Example 5.3 Let $X=L^{p}([0,1])$. If a function $g:[0,1] \rightarrow \mathbb{C}^{*}$ - is bounded, then the linear operator $\mathfrak{G}$ of multiplication by $g$ is an isomorphism of $L^{p}([0,1])$. Since the function $g:[0,1] \rightarrow \mathbb{C}^{*}$ defined by equality (5.7) is continuous and hence bounded, we infer that the corresponding operator $\mathfrak{G}^{\mathfrak{s}}$ is an isomorphism of $L^{p}([0,1])$.

Example 5.4 Let $X=\mathrm{V}_{\alpha}$ for some $\alpha \in(0,1]$. Assume that both functions $h$ and $\widehat{h}$ belong to the space $c^{\alpha}([0,1])$. Then both equations (5.1) and (5.2) generate a $C_{0^{-}}$ semigroup on $\mathrm{V}_{\alpha}$ and, by Proposition A.1, the operator $\mathfrak{C}$ is an isomorphism.

Now we shall prove the theorems analogous to 3.1 and 4.1 in the case if $h$ is not constant.

Theorem 5.5 Let $h:[0,1] \rightarrow \mathbb{C}$ be the continuous function satisfying the following condition

(H) There exist numbers $\delta>0$ and $\lambda>-\frac{1}{p}$ such that

$$
\operatorname{Re} h(x)>\lambda \quad \text { for } x \in[0, \delta] \text {. }
$$

Then the $C_{0}$-semigroup generated by equation (5.1) on the space $L^{p}$ is chaotic. 
Remark 5.6 It is obvious, that if a continuous function $h:[0,1] \rightarrow \mathbb{C}$ satisfies conditions (1) and (2) from the beginning of this section, then $h$ also satisfies our assumption $(\mathrm{H})$. Hence our theorem 5.5 is stronger, than that in [16]

Proof Let us choose $\lambda$ and $\delta>0$ so that assumption (H) holds. Hence, the function $\rho:[0,1] \rightarrow \mathbb{C}$ defined by

$$
\rho(x)=\exp \left(-\int_{x}^{1} \frac{h(s)-\lambda}{s} d s\right), \quad x \in[0,1],
$$

is well-defined, continuous and $\rho(0)=0$. In particular, $\rho$ is a bounded function. Therefore, the multiplication by $\rho$ defines a bounded, injective linear operator $\mathbf{R}$ on the space $L^{p}$. It is easy to verify that if $u$ is a solution to problem (2.1), then $\tilde{u}$ defined by the following formula

$$
\widetilde{u}(t, x)=\rho(x) u(t, x)
$$

is the solution to problem (5.1). Moreover, the diagram

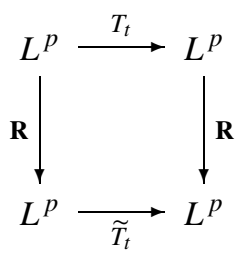

where $\left\{\widetilde{T}_{t}\right\}_{t \geq 0}$, resp. $\left\{T_{t}\right\}_{t \geq 0}$, is the dynamical system generated by (5.1), resp. (2.1), is commuting.

To complete the proof it is sufficient to show that the range of $\mathbf{R}$, i.e. the set $\mathbf{R}\left(L^{p}\right)$, is dense in $L^{p}$. To prove this let us notice that for every $\varepsilon>0$ there exits $\delta>0$ such that $\rho(x) \in\left[\delta, \frac{1}{\delta}\right]$ for all $x \in[\varepsilon, 1]$.

Take now $u \in L^{p}$ and let define an $L^{p}(0,1)$-valued sequence $\left(u_{n}\right)_{n=1}^{\infty}$ by

$$
u_{n}(x)= \begin{cases}u(x), & \text { if } x \in\left(\frac{1}{n}, 1\right] \\ 0, & \text { if } x \in\left[0, \frac{1}{n}\right]\end{cases}
$$

Clearly $\lim _{n \rightarrow \infty}\left\|u_{n}-u\right\|_{L^{p}}=0$ and by the property of $\rho$ the functions $\frac{u_{n}}{\rho}$ belong to $L^{p}$. Therefore, $u_{n}=\mathbf{R}\left(\frac{u_{n}}{\rho}\right) \in \mathbf{R}\left(L^{p}\right)$ what completes the proof.

The above Theorem 5.5 can be generalized to the framework analogous to the one studied in [16].

Theorem 5.7 Assume that $\alpha \in(0,1]$. Assume that $h:(0,1] \rightarrow \mathbb{C}$ is a Lebesgue measurable function satisfying the following condition.

(H2) There exist a real number $\lambda$ such that $\lambda>\alpha$ that

$$
\int_{0}^{1}\left|\frac{\operatorname{Re} h(x)-\lambda}{x}\right| d x<\infty .
$$

Then the $C_{0}$ semigroup generated by equation (5.1) on the space $V_{\alpha}$ is chaotic. 
Proof From the condition (5.10) it follows that

$$
\int_{0}^{1}\left|\frac{\operatorname{Re} h(x)-\lambda}{x}\right| d x<\infty .
$$

Define next a function $\rho:[0,1] \rightarrow \mathbb{C}$ by the following formula

$$
\rho(x)=\exp \left(-\int_{x}^{1} \frac{h(s)-\lambda}{s} d s\right), \quad x \in[0,1] .
$$

It follows from the above formula that

$$
\frac{1}{\rho(x)}=\exp \left(\int_{x}^{1} \frac{h(s)-\lambda}{s} d s\right), \quad x \in[0,1]
$$

and hence, in view of (5.11), both functions $\rho$ and $\frac{1}{\rho}$ are bounded and Lipschitz on $[0,1]$. Hence by Proposition A.1 the operator $\mathbf{R}$ of multiplication by $\rho$ is an isomorphism of $V_{\alpha}$. We can complete the proof by following the same argument as in proof of Theorem 5.5.

Acknowledgements Part of the work was done during a visit of the 2 nd named authour to the University of York. He wishes to thank the Department of Mathematics the University of York for hospitality. The authours would like to thank an anonymous referee for pointing out the work [16] by Takeo.

Open Access This article is distributed under the terms of the Creative Commons Attribution License which permits any use, distribution, and reproduction in any medium, provided the original author(s) and the source are credited.

\section{Appendix A: Multiplication operators in the space $V_{\alpha}$}

We will prove the following result.

Proposition A.1 Assume that $\alpha \in(0,1]$. Then for all $g \in c^{\alpha}([0,1])$ and $u \in \mathrm{V}_{\alpha}$, the product gu belongs to $\mathrm{V}_{\alpha}$. Moreover,

$$
H_{r, \alpha}(g u) \leq|g|_{L^{\infty}(0,1)} H_{r, \alpha}(u)+H_{r, \alpha}(g)|u|_{L^{\infty}(0,1)} .
$$

Proof Take and fix $g \in c^{\alpha}([0,1])$ and $u \in \mathrm{V}_{\alpha}$. Obviously $g u \in L^{\infty}(0,1)$ and

$$
|g u|_{L^{\infty}} \leq|g|_{L^{\infty}}|u|_{L^{\infty}} .
$$

Moreover, for $x, y \in[0,1]$ such that $x \neq y$ :

$$
\frac{|g(x) u(x)-g(y) u(y)|}{|x-y|^{\alpha}} \leq|g(x)| \frac{|u(x)-u(y)|}{|x-y|^{\alpha}}+\frac{|g(x)-g(y)|}{|x-y|^{\alpha}}|u(y)| .
$$

Hence, for every $r \in(0,1]$, we have

$$
H_{r, \alpha}(g u) \leq|g|_{L^{\infty}} H_{r, \alpha}(u)+H_{r, \alpha}(g)|u|_{L^{\infty}} .
$$


In particular, taking $r=1$, we deduce inequality (A.1). It remains to prove that $g u \in$ $V_{\alpha}$. But, taking the $\limsup _{r \rightarrow 0}$ in (A.2) we infer that

$$
\limsup _{r \rightarrow 0} H_{r, \alpha}(g u) \leq|g|_{L^{\infty}} \limsup _{r \rightarrow 0} H_{r, \alpha}(u)+\limsup _{r \rightarrow 0} H_{r, \alpha}(g)|u|_{L^{\infty}} .
$$

The last inequality concludes the proof.

Remark A.2 As far as we are aware Proposition A.1 is known in the case of spaces $C^{\alpha}$, see for instance [1]. Another proof of Proposition A.1 would be to use this result together with the fact that the spaces $c^{\alpha}$ and $V_{\alpha}$ are closures in the $C^{\alpha}$ space of appropriate spaces of smooth functions.

\section{References}

1. Appell, J., Zabrejko, P.P.: Nonlinear superposition operators. Cambridge Tracts in Mathematics, vol. 95. Cambridge University Press, Cambridge (1990)

2. Brzeźniak, Z., Dawidowicz, A.L.: On periodic solutions to the von Foerster-Lasota equation. Semigroup Forum 78(1), 118-137 (2009)

3. Dawidowicz, A.L.: On the existence of an invariant measure for a quasi-linear partial differential equation. Zesz. Nauk. Uniw. Jagiell., Pr. Mat. 23, 117-123 (1982)

4. Dawidowicz, A.L.: On the existence of an invariant measure for the dynamical system generated by partial differential equation. Ann. Pol. Math. XLI, 129-137 (1983)

5. Dawidowicz, A.L., Haribash, N.: On the periodic solutions of von Foerster type equation. Univ. Iagellonicae Acta Math. 37, 321-324 (1999)

6. Dawidowicz, A.L., Haribash, N.: On the dense trajectory of Lasota equation. Univ. Iagellonicae Acta Math. 43, 61-66 (2005)

7. Desch, W., Schappacher, W., Webb, G.F.: Hypercyclic and chaotic semigroups of linear operators. Ergod. Theory Dyn. Syst. 17(4), 793-819 (1997)

8. Devaney, R.L.: An Introduction to Chaotic Dynamical Systems, 2nd edn. Addison-Wesley Studies in Nonlinearity. Addison-Wesley, Redwood City (1989)

9. Evans, L.C.: Partial Differential Equations. American Mathematical Society, Providence (1998)

10. Hale, J.K.: Asymptotic Behavior of Dissipative Systems. Mathematical Surveys and Monographs, vol. 25. American Mathematical Society, Providence (1988)

11. Horváth, M.: Some saturation theorems for classical orthogonal expansions. II. Ann. Math. Hung. 58(1-2), 157-191 (1991)

12. Lasota, A., Pianigiani, G.: Invariant measures on topological spaces. Boll. Unione Mat. Ital. 5(15-B), 592-603 (1977)

13. Lasota, A., Szarek, T.: Dimension of measures invariant with respect to Ważewska partial differential equations. J. Differ. Equ. 196(2), 448-465 (2004)

14. Łoskot, K.: Turbulent solutions of first order partial differential equation. J. Differ. Equ. 58(1), 1-14 (1985)

15. Rudnicki, R.: Invariant measures for the flow of a first order partial differential equation. Ergod. Theory Dyn. Syst. 5(3), 437-443 (1985)

16. Takeo, F.: Chaos and hypercyclity for solution semigroup to some partial differehtial equations. Nonlinear Anal. 63, e1943-e1953 (2005)

17. Yosida, K.: Functional Analysis. Classics in Mathematics. Springer, Berlin (1995). Reprint of the 6th edn. (1980) 